\section{Animals and Their Unicellular Ancestors}

Jordi Paps, Universitat de Barcelona, Barcelona, Spain

Iñaki Ruiz-Trillo, Universitat de Barcelona, Barcelona, Spain

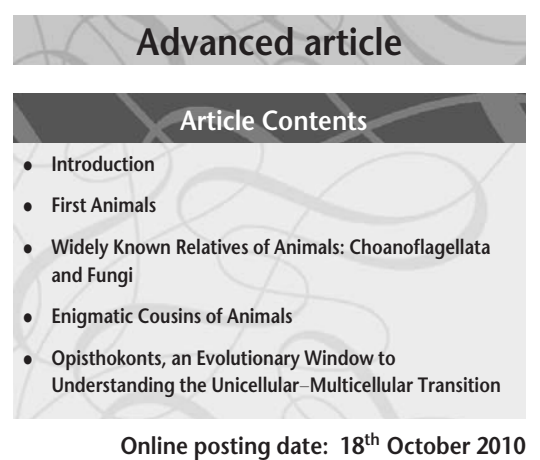

Monera (which comprises the prokaryotic bacteria and archeae).

Thanks to many advances in molecular techniques, this rather simplistic view is being largely improved. First came Carl Woese's proposal (Woese and Fox, 1977) to divide the Tree of Life into three domains: two prokaryotic domains, Archaea and Bacteria, and an eukaryotic domain (Eukaryota). From then on, a myriad of molecular phylogenies improved that scheme, dividing the Eukaryota into a few major groups. Cavalier-Smith (2002) grouped those eukaryotic supergroups into two major clades: the unikonts and the bikonts. The name 'Unikonta' (from Greek, uni meaning 'single' and kontos meaning 'pole', referring to the flagellum) refers to the structure of kinetids, that is, the flagellar apparatus composed of one or two basal bodies (centrioles plus their related microtubules) in conjunction with their associated cilia/flagella. Cavalier-Smith has claimed (2002) that the last common ancestor of Unikonta had only one centriole and one cilium per kinetid. Unikonts include Amoebozoa and Opisthokonta, the clade that includes animals and fungi. The unikont-bikont division of eukaryotes is still highly contentious, because, even if considered derived states, many current unikonts have two centrioles or two cilia. In any case, molecular phylogenies (Ruiz-Trillo et al., 2008), different molecular synapomorphies (Richards and Cavalier-Smith, 2005) and recent analyses on rare genomic changes (Rogozin et al., 2009) seem to corroborate the alliance of opisthokonts with amoebozoans. See also: Protozoan Evolution and Phylogeny

Embedded among the unikonts, 'Opisthokonta' (from Greek, opisthō means 'posterior'; Cavalier-Smith, 1987) are unikonts with (1) a single posterior flagellum (in at least one vital phase sometimes secondarily lost) with a pair of centrioles and (2) exhibiting mitochondria with flat cristae (compartments outlined by the mitochondrion inner membrane). Opisthokonta comprises animals, plus fungi and several unicellular groups, such as the choanoflagellates (Figure 1). This is why opisthokonts are sometimes informally named as the 'Metazoa/Fungi clade'. The monophyly of opisthokonts has been confirmed by molecular phylogenies (Lang et al., 2002; Ruiz-Trillo et al., 2004, 2008), an approximately 12-amino acid insertion present in the translation elongation factor 1-alpha (EF1$\alpha$; Baldauf and Palmer, 1993) and the presence of a 


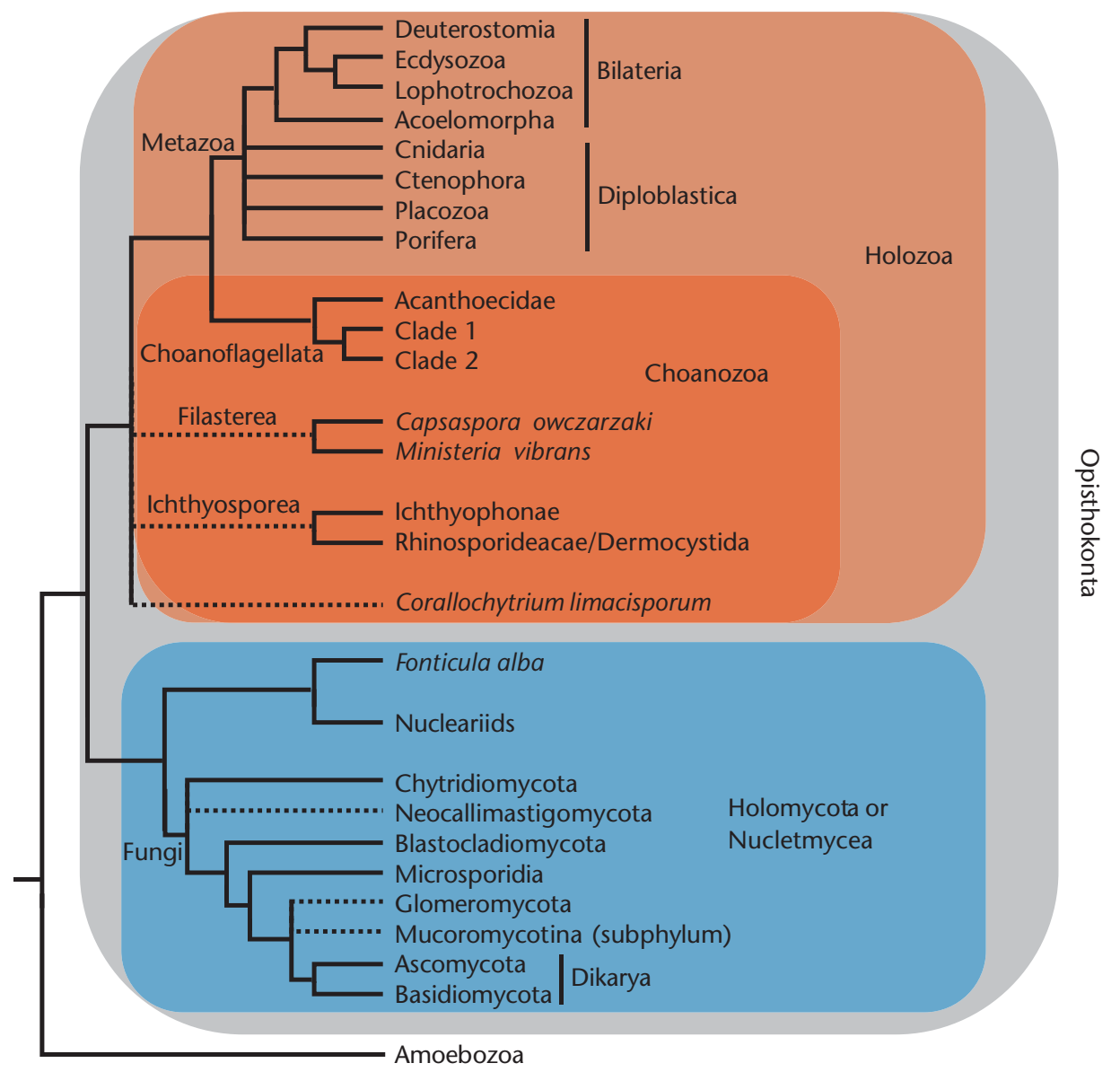

Figure 1 Phylogeny of the Opisthokonta. Summary of the phylogenetic relationships of the opisthokonts, based on recent molecular phylogenies. Amoebozoans are sister group to Opisthokonta, together forming the Unikonta. Shaded boxes remark widely accepted supergroups. See text for further discussion.

haloarchaeal type tyrosyl-transfer ribonucleic acid (tRNA) synthetase (Huang et al., 2005).

\section{First Animals}

'Animals' were defined by Cavalier-Smith (1998) as phagotrophic multicellular eukaryotes with a connective tissue, located between two dissimilar epithelia (except in Mesozoa and Placozoa) and composed of collagen (Figure 2a). Later, Adl et al. (2005) extended this definition by adding traits such as osmotrophic nutrition, absence of cell wall, presence of intercellular junctions holding cells and sexual reproduction with production of an egg cell that is fertilised by a smaller, often monociliated, sperm cell. There are a million and half described animal species, grouped in around 35 phyla. However, some estimations suggest that the real number is probably greater than 11 million species. See also: Diversity of Life

Animal relationships have been explored since Haeckel coined the term phylogeny, one century and a half ago, and modern molecular phylogenetics has presented a widely accepted 'new animal phylogeny' (Aguinaldo et al., 1997; Halanych, 2004; Halanych et al., 1995). This novel scheme distributes the animals into two big groups, Diploblastica (animals with radial symmetry) and Bilateria (animals with bilaterian symmetry). The new rearrangement made by molecular systematics mostly affected the relationships within bilaterians, defining novel groups such as Lophotrochozoa or Ecdysozoa (Aguinaldo et al., 1997; Halanych et al., 1995). It is widely accepted that diploblasts are the earliest-branching Metazoa, embracing phyla such as Porifera (sponges), Cnidaria (sea anemones and jellyfishes), Ctenophora (comb jellies) and the puzzling Placozoa (with only a single species described, Trichoplax adhaerens). Whereas the position of Cnidaria seems to be settled as a sister group to bilaterians (Halanych, 2004), the remaining deep animal relationships are still problematic and questions about the monophyly of sponges or the position of sponges, ctenophores and placozoans have not been cleared yet.

Early phylogenetic studies favoured the paraphyletic status of 'Porifera', with Hexactinellida (glass sponges), then Demospongiae (spongin fibres) and then Calcarea 
(calcareous sponges) splitting off in order from the metazoan last common ancestor (Borchiellini et al., 2001). Recent studies have failed to provide a definitive answer on to the Porifera status, because some trees show Porifera as a monophyletic assemblage (Pick et al., 2010) whereas others support their paraphyly (Hejnol et al., 2009; Sperling et al., 2009). However, most of these phylogenomic studies agree in the sister group relationship between hexactinellids and demosponges.

The morphologically simple 'Placozoa' have also been candidates to be direct descendants from the metazoan last common ancestor (Schierwater et al., 2009). However, molecular phylogenies have shown placozoans in every possible position among the diploblasts, from sister group to Cnidaria (Bridge et al., 1995), to basal diploblasts (Dellaporta et al., 2006; Schierwater et al., 2009), to an intermediate position between sponges and cnidarians (Srivastava et al., 2008). Finally, the diploblast phylum 'Ctenophora' has recently been shown to be the earliestbranching metazoans (Dunn et al., 2008; Hejnol et al., 2009). This position is highly controversial and whether is real or a result of an artefact caused by low taxon sampling or long-branch attraction (Pick et al., 2010) awaits additional data.

\section{Widely Known Relatives of Animals: Choanoflagellata and Fungi}

The other major group of opisthokonts is the Fungi. 'Fungi' are defined as heterotrophic organisms, with unicellular or multicellular species, with vegetative and spore cells, often with cell walls of chitin and beta-glucan (at least in spores) and without phagocytosis (Cavalier-Smith, 1998). They display multinucleate hyphae, an alpha-aminoadipate (AAA) lysine biosynthesis pathway (although this pathway may present in other eukaryotes, see Torruella et al., 2009), mitochondria and peroxisomes but lack other plastids (Adl et al., 2005). The Fungi share some traits with metazoans as the presence of chitin, storage of glycogen and mitochondrial codon UGA encoding tryptophan; both have spores or gametes with a single posterior flagellum, but only the fungi chytrids have kept this character (Cavalier-Smith, 1987). Around 75000 species of fungi have been described, but the estimates suggest there are above one million species. Their diversity range from the simple yeast or molds (i.e. Saccharomyces or Penicillium) to the more eye-catching mushrooms (Figure $\mathbf{2 b}$ ), including a huge array of plant pathogens (such as the rice blast Magnaporthe grisea) or organisms of medical interest such as Candida, Aspergillus or Cryptococcus. A recent thorough revision, heavily based in molecular data, has reorganised all the fungi groups into seven phyla (Hibbett et al., 2007; see Figure 1 for phyla names). The most remarkable modifications affect the first splitting fungi, the two traditional phyla Chytridiomycota and Zygomycota, which currently are recognised as polyphyletic groups
(James et al., 2006). Recent multigenic (James et al., 2006) and phylogenomics studies (Liu et al., 2009) show Chytridiomycota (sensu Hibbett et al., 2007) as the most basal fungi, together or followed by Neocallimastigomycota (Figure 1). See also: Diversity of Life

The other major, and well-known, opisthokont lineage besides fungi and animals is the 'Choanoflagellata'. Choanoflagellates comprise around 140 species of mostly marine heterotrophic, flagellate single-celled organisms, although some of them are able to form simple colonies (i.e. Proterospongia, see King, 2005). They present an eggshaped cell 3-10 $\mu \mathrm{m}$ in diameter and bear a single posterior flagellum enclosed by a collar of thin microvilli. The idea that choanoflagellates are close relatives of animals was long ago posited on the basis of the striking resemblance between choanoflagellates and the collared cells (choanocytes) of sponges (James-Clark, 1866). In fact, recent molecular phylogenies have confirmed that choanoflagellates are indeed the sister group to Metazoa (Lang et al., 2002; Carr et al., 2008; Ruiz-Trillo et al., 2008). Choanoflagellates external morphology is remarkably diverse, with outer coverings ranging from very simple ones to incredibly intricate coats. Actually, their external diversity is the basis of their traditional classification into three families: Codosigidae, Salpingoecidae and Acanthoecidae. The Codosigidae are the 'naked' choanoflagellates, they only have the cell membrane as an outer covering. The Salpingoecidae have a flask-shaped membrane or 'theca', whereas the Acanthoecidae have beautiful silica baskets or 'lorica'. A recent molecular study analysing four genes for 17 choanoflagellates species (Carr et al., 2008) confirmed the existence of acanthoecids, while rejecting the monophyly of the other two families. Owing to their pivotal position as the closest unicellular relative to animals, our knowledge on them is relatively good compared with other no-parasite protists, with a genome sequence already published (King et al., 2008), another in progress (Ruiz-Trillo et al., 2007) and some expressed sequence tags (ESTs) projects done (King et al., 2003; Ruiz-Trillo et al., 2008).

\section{Enigmatic Cousins of Animals}

In addition to the major opisthokonts groups explored earlier (animals, fungi and choanoflagellates), recent molecular data have shown that Opisthokonta includes other poorly known single-celled lineages, such as ichthyosporeans, filastereans, nucleariids, Corallochytrium limacisporum, and the most recent addition, the slime mold Fonticula alba. Later, we describe each of those groups.

'Ichthyosporea' (Cavalier-Smith, 1998) was originally defined as the DRIP group (by the acronym of Dermocystidium, rossete agent, Ichthyophonus and Psorospermium), a node-based clade closely related to animals and choanoflagellates (Herr et al., 1999). Later their name was amended to Mesomycetozoea (Mendoza et al., 2002), based on the clade name 'mesomycetozoa' (Herr et al., 


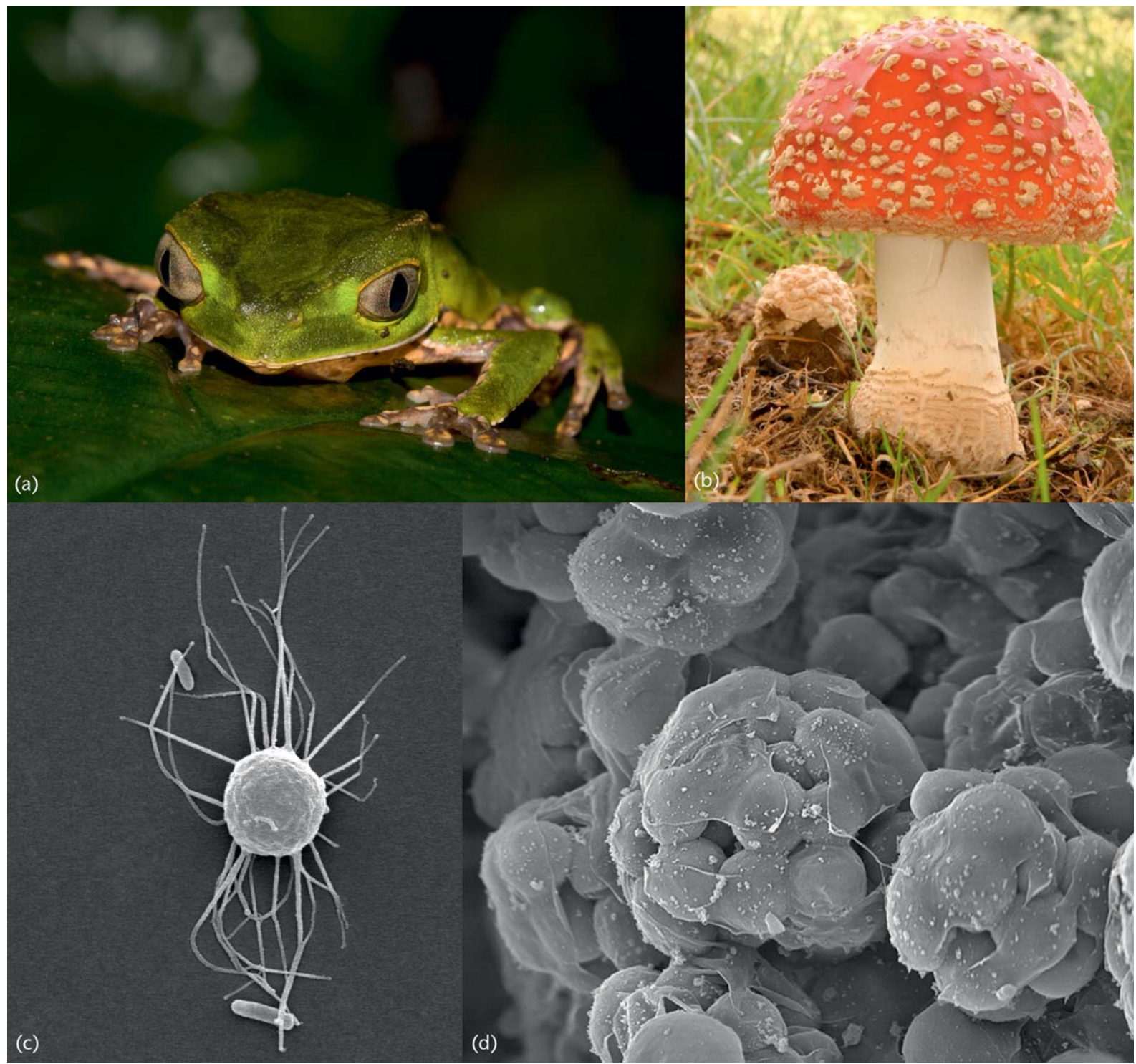

Figure 2 Pictures representatives of different Opisthokonta. (a) Picture of the frog Phyllomedusa vaillantii. Courtesy of Carlos Palacín and Alejandro de Mendoza. (b) Picture of the fungi Amanita muscaria. (c) Scanning microscopy picture of the filasterean Ministeria vibrans. (d) Scanning microscopy picture of the ichthyosporean Creolimax fragrantissima in colonial stage. Courtesy of Arnau Sebé-Pedrós.

1999) that refers to their evolutionary position between fungi and animals (meso means 'middle', myceto stands for fungi and zoa for animals). However, probably to avoid confusion with Mycetozoa, Ichthyosporea seems to be nowadays the most accepted name for this clade. Ichthyosporea are single-celled organisms, with flat cristae like other opisthokonts, and some show colonial forms (Figure 2d). Most are animal parasites or endosymbionts, although there are some free-living and saprotrophic species (Adl et al., 2005). Ichthyosporeans can be divided into two groups, the human parasitic Rhinosporideacae and the Ichthyophonae. Some Rhinosporideacae (Mendoza et al., 2002; Dermocystida in Cavalier-Smith, 1998) show a posterior flagellum, and when inside their hosts, they exhibit sphere-shaped cells with several endospores that are eventually released. Ichthyophonae (Mendoza et al., 2002; Ichthyophonida Cavalier-Smith, 1998), on the contrary, includes parasites of fish or aquatic arthropods. Several of them show amoeboid cells typical of the group, although in others, amoeboid cells are absent or have not been detected yet. None of them shows flagellum, albeit a contentious monoflagellated phase is reported in Pseudoperkinsus tapetis. Interestingly, some ichthyosporeans have the capacity to form microcolonies as part of the cell cycle (Ruiz-Trillo et al., 2007).

Early molecular trees related ichthyosporeans to choanoflagellates (Herr et al., 1999; Mendoza et al., 2002), but later when filastereans (see discussion later) started to be 
added to phylogenetic or phylogenomic analyses, Ichthyosporea was shown as sister group to filastereans or to animals, choanoflagellates and filastereans (Carr et al., 2008; Ruiz-Trillo et al., 2008; Shalchian-Tabrizi et al., 2008; Steenkamp et al., 2006). Aphelidea (Gromov, 2000) are intracellular parasites of algae of undetermined phylogenetic position with an intricate life cycle that starts with an amoeboid cell invading the algae and finishes when cell division releases flagellate or amoeboid cells. Although aphelids have been proposed as members of Ichthyosporea (Shalchian-Tabrizi et al., 2008), the lack of molecular data for them keeps their position unsettled.

'Filasterea' is a newly defined group (Shalchian-Tabrizi et al., 2008), mainly based on molecular data, which currently comprises two species, Capsaspora owczarzaki (ATCC 30864) and Ministeria vibrans. Both organisms are unicellular, lack flagella and display flat cristae and long filopodia emerging from their membranes. Capsaspora amoeboid-like cells $(3.0-7.0 \mu \mathrm{m})$ were isolated from the pulmonate snail Biomphalaria glabrata (Stibbs et al., 1979), where they seem to feed from the larvae of the trematode Schistosoma mansoni that parasite the snail. Capsaspora is, thus, a symbiont of B. glabrata and was first named as Nuclearia sp. and considered a member of the nucleariids (Amaral-Zettler et al., 2001). Further morphological and phylogenetic analyses showed that $C$. owczarzaki is not a nucleariid but an independent opisthokont lineage, closely related to ichthyosporeans (Ruiz-Trillo et al., 2004). Ministeria (Tong, 1997) are free-living marine microorganisms, around $5 \mu \mathrm{m}$ in diameter (Figure $2 \mathrm{c}$ ). Some single-gene or multi-gene phylogenies related it to animals (CavalierSmith and Chao, 2003). Later analyses including data from both Capsaspora and Ministeria showed their sister group relationship and placed them closer to animals and choanoflagellates, forming the group Filozoa (Brown et al., 2009; Shalchian-Tabrizi et al., 2008).

'Corallochytrea' comprises a single species, C. limacisporum (Raghu-Kumar, 1987), a marine saprotrophic organism, 4.5-20 $\mu \mathrm{m}$ diameter, found in coral reefs in the Indian Ocean. During their life cycle, they undergo binary fissions and produce several amoeboid cells. The presence of flagellum has been reported. They were first classified as a kind of Fungi (as a Thraustochytrid, Raghu-Kumar, 1987), but early molecular phylogenies placed it close to choanoflagellates (Cavalier-Smith and Chao, 2003; Mendoza et al., 2002) and later multigenic analyses pointed to a relationship with ichthyosporeans (Carr et al., 2008; Ruiz-Trillo et al., 2004; Steenkamp et al., 2006). Unfortunately, there is no living culture available to researchers; therefore, its specific phylogenetic position within the opisthokonts may be unclear for some time more.

'Nucleariidae' (Cavalier-Smith, 1993) comprise several free-living, heterotrophic amoeboids. Besides their extending hyaline filopodia, nucleariids have either discoidal-shaped or flattened mitochondrial cristae. They typically have a spherical floating form and an amoeboid form when adhered to a substrate. Molecular phylogenies have strongly shown that nucleariids are the sister group to
Fungi (Liu et al., 2009; Ruiz-Trillo et al., 2004; Steenkamp et al., 2006).

Finally, the most recent addition to the opisthokonts is the cellular slime mold ' $F$. alba' (Brown et al., 2009). This species, the only on its genus, is an amoeboid protist that forms a multicellular fruiting body by aggregation of several individuals (Worley et al., 1979). Molecular phylogenies have shown that $F$. alba is the sister group to nucleariids.

\section{Opisthokonts, an Evolutionary Window to Understanding the Unicellular-Multicellular Transition}

Our current phylogenetic framework of the opisthokonts splits them into two major branches: one containing Fungi and their single-celled relatives, named as Nucletmycea (Brown et al., 2009) or Holomycota (Liu et al., 2009), and another one containing Metazoa and their unicellular relatives, known as Holozoa (see Figure 1; Lang et al., 2002). Moreover, the single-celled holozoans, that is, the closest animal relatives, are also known as choanozoans.

Interestingly, within the opisthokonts, there are currently five types of multicellular organisation: animal, fungal, aggregative colonial, 'syncitial type' colonial and aggregative fruiting bodies. Animal multicellularity is the product of embryogenesis, where a single cell develops into various differentiated cells and tissues, whereas Fungi have mycelia, which are interconnected, walled filamentous cells, which may display syncitial features. Choanoflagellates and $F$. alba can form colonies or fruiting bodies, respectively, by aggregation of several individuals, whereas some ichthyosporeans develop 'syncitial' type microcolonies.

Thus, the opisthokonts offer a unique evolutionary window among eukaryotic supergroups to understand multicellular origins. In this regard, the single-celled members of Holomycota, nucleariids and $F$. alba, are crucial to understanding the origin of fungal multicellularity, whereas the single-celled members of Holozoa, that is filastereans, ichthyosporeans, choanoflagellates and Corallochytrium, appear to be critical to understanding the origin of animal multicellularity. The importance of animal relatives to understanding the origin of animals has often been neglected, with most research efforts focusing on the study of basal metazoans, such as cnidarians, poriferans and placozoans. Studies of those basal animals have already unravelled important insights into the origin of Metazoa, and now researchers have complete genome sequence of two cnidarians (Hydra magnipapillata and Nematostella vectensis), one poriferan (Amphimedon queenslandica) and the placozoan Trichoplax adhaerens (Table 1). However, only a single genome of an animal relative (that of the choanoflagellate Monosiga brevicollis) has, so far, been published (King et al., 2008). That genome showed us that genomic comparisons between basal 
Table 1 Current available genomic data from Opisthokonta in NCBI

\begin{tabular}{lccc}
\hline Organisms & Genomes completed & Genomes in progress & ESTs reads \\
\hline Metazoa & & 58 & $20 \times 10^{6}$ \\
$\quad$ Bilaterian invertebrates & 24 & 0 & $640 \times 10^{3}$ \\
$\quad$ Cnidaria & 2 & 0 & $51 \times 10^{3}$ \\
$\quad$ Ctenophora & 0 & 0 & $11 \times 10^{3}$ \\
$\quad$ Placozoa & 1 & 1 & $95 \times 10^{3}$ \\
$\quad$ Porifera & 1 & 1 & $106 \times 10^{3}$ \\
Choanoflagellata & & 1 & $9 \times 10^{3}$ \\
Filasterea & 0 & 1 & 68 \\
$\quad$ Capsaspora owczarzaki & 0 & 1 & $8 \times 10^{3}$ \\
$\quad$ Ministeria vibrans & 0 & 1 & - \\
Ichthyosporea & 0 & 1 & $4 \times 10^{3}$ \\
$\quad$ Sphaeroforma arctica & 0 & 50 & $3.5 \times 10^{6}$ \\
$\quad$ Creolimax fragrantissima & & 0 & - \\
$\quad$ Amoebidium parasiticum & 200 & 1 & \\
Fungi Nucleariids & 0 & 0 & $10^{6}$ \\
Fonticula alba & & & \\
\hline
\end{tabular}

Source: Extracted from NCBI web page (http://www.ncbi.nlm.nih.gov/).

animals and their current unicellular relatives will provide significant insights into the emergence of animals from their protistan ancestors.

However, if one looks at the opisthokont phylogenetic framework (see Figure 1), it is clear that only by incorporating additional genomic data from the other animal relatives, such as ichthyosporean, filastereans and Corallochytrium, in the comparative analyses, we will be able to fully disentangle the 'metazoan genetic starter kit'. This is actually the goal of the UNICORN initiative, recently funded by the National Human Genome Research Institute (NHGRI) and being performed at the Broad Institute at MIT and Harvard (Ruiz-Trillo et al., 2007). UNICORN is obtaining the genome sequence of several holozoans and holomycots, with the aim to better understand the origins of both fungi and animals. A first look at what this data can show us is provided by the analysis of the integrin-mediated signalling and adhesion machinery that conclusively shows that choanoflagellates (and fungi) have suffered some lineage-specific gene losses of this important machinery (Sebé-Pedrós et al., 2010). This should be taken as a cautionary tale, because gene loss is undoubtedly an important, and sometimes neglected, player in evolutionary history and that broad taxonomic sampling is critical in comparative genomic analyses. Thus, we envision, and hope, that animal unicellular relatives, such as Capsaspora, Ministeria or ichthyosporeans, will not only offer us important insights into animal origins but also no longer be neglected by researchers.

\section{References}

Adl S, Simpson A, Farmer M et al. (2005) The new higher level classification of eukaryotes with emphasis on the taxonomy of protists. Journal of Eukaryotic Microbiology 52: 399-451.
Aguinaldo AM, Turbeville JM, Linford LS et al. (1997) Evidence for a clade of nematodes, arthropods and other moulting animals. Nature 387: 489-493.

Amaral-Zettler LA, Nerad TA, O'Kelly CJ et al. (2001) The nucleariid amoebae: more protists at the animal-fungal boundary. Journal of Eukaryotic Microbiology 48: 293-297.

Baldauf S and Palmer J (1993) Animals and fungi are each other's closest relatives: congruent evidence from multiple proteins. Proceedings of the National Academy of Sciences of the USA 90: 11558-11562.

Borchiellini C, Manuel M, Alivon E et al. (2001) Sponge paraphyly and the origin of Metazoa. Journal of Evolutionary Biology 14: 171-179.

Bridge D, Cunningham CW, DeSalle R et al. (1995) Classlevel relationships in the phylum Cnidaria: molecular and morphological evidence. Molecular Biology and Evolution 12: 679-689.

Brown M, Spiegel F and Silberman J (2009) Phylogeny of the "forgotten" cellular slime mold, Fonticula alba, reveals a key evolutionary branch within Opisthokonta. Molecular Biology and Evolution 26: 2699-2709.

Carr M, Leadbeater B, Hassan R et al. (2008) Molecular phylogeny of choanoflagellates, the sister group to Metazoa. Proceedings of the National Academy of Sciences of the USA 105: 16641-16646.

Cavalier-Smith T (1987) The origin of fungi and pseudofungi. In: Rayner ADM, Brasier CM and Moore D (eds) Evolutionary Biology of the Fungi, pp. 339-353. Cambridge, UK: Cambridge University Press.

Cavalier-Smith T (1993) Kingdom protozoa and its 18 phyla. Microbiology and Molecular Biology Reviews 57: 953994.

Cavalier-Smith T (1998) A revised six-kingdom system of life. Biological Reviews of the Cambridge Philosophical Society (London) 73: 203-266.

Cavalier-Smith T (2002) The phagotrophic origin of eukaryotes and phylogenetic classification of Protozoa. International 
Journal of Systematic and Evolutionary Microbiology 52: 297-354.

Cavalier-Smith T and Chao E (2003) Phylogeny of choanozoa, apusozoa, and other protozoa and early eukaryote megaevolution. Journal of Molecular Evolution 56: 540-563.

Dellaporta SL, Xu A, Sagasser S et al. (2006) Mitochondrial genome of Trichoplax adhaerens supports Placozoa as the basal lower metazoan phylum. Proceedings of the National Academy of Sciences of the USA 103: 8751-8756.

Dunn C, Hejnol A, Matus D et al. (2008) Broad phylogenomic sampling improves resolution of the animal tree of life. Nature 452: $745-749$.

Gromov BV (2000) Algal parasites of the genera Aphelidium, Amoeboaphelidium, and Pseudoaphelidium from the Cienkovski's "Monadinea" group as representatives of a new class. Zoologichesky Zhurnal 79: 517-525.

Halanych KM (2004) The new view of animal phylogeny. Annual Review of Ecology, Evolution, and Systematics 35: 229-256.

Halanych KM, Bacheller JD, Aguinaldo AM et al. (1995) Evidence from $18 \mathrm{~S}$ ribosomal DNA that the lophophorates are protostome animals. Science 267: 1641-1643.

Hejnol A, Obst M, Stamatakis A et al. (2009) Assessing the root of bilaterian animals with scalable phylogenomic methods. Proceedings. Biological Sciences/Royal Society B 276: 4261-4270.

Herr RA, Ajello L, Taylor JW et al. (1999) Phylogenetic analysis of Rhinosporidium seeberi's $18 \mathrm{~S}$ small-subunit ribosomal DNA groups this pathogen among members of the protoctistan Mesomycetozoa clade. Journal of Clinical Microbiology 37: 2750-2754.

Hibbett DS, Binder M, Bischoff JF et al. (2007) A higher-level phylogenetic classification of the Fungi. Mycological Research 111: 509-547.

Huang J, Xu Y and Gogarten J (2005) The presence of a haloarchaeal type tyrosyl-tRNA synthetase marks the opisthokonts as monophyletic. Molecular Biology and Evolution 22: 2142-2146.

James T, Kauff F, Schoch C et al. (2006) Reconstructing the early evolution of Fungi using a six-gene phylogeny. Nature 443: $818-822$.

James-Clark H (1866) Note on the infusoria flagellata and the spongiae ciliatae. American Journal of Science 1: 113-114.

King N (2005) Choanoflagellates. Current Biology 15: R113-R114.

King N, Hittinger CT and Carroll SB (2003) Evolution of key cell signaling and adhesion protein families predates animal origins. Science 301: 361-363.

King N, Westbrook M, Young S et al. (2008) The genome of the choanoflagellate Monosiga brevicollis and the origin of metazoans. Nature 451: 783-788.

Lang BF, O'Kelly C, Nerad T et al. (2002) The closest unicellular relatives of animals. Current Biology 12: 1773-1778.

Liu Y, Steenkamp E, Brinkmann H et al. (2009) Phylogenomic analyses predict sistergroup relationship of nucleariids and fungi and paraphyly of zygomycetes with significant support. BMC Evolutionary Biology 9: 272.

Mendoza L, Taylor JW and Ajello L (2002) The class mesomycetozoea: a heterogeneous group of microorganisms at the animal-fungal boundary. Annual Review of Microbiology 56: $315-344$.
Pick K, Philippe H, Schreiber F et al. (2010) Improved phylogenomic taxon sampling noticeably affects non-bilaterian relationships. Molecular Biology and Evolution 27(9): $1983-$ 1987.

Raghu-Kumar S (1987) Occurrence of the thraustochytrid, Corallochytrium limacisporum gen. et sp. nov. in the coral reef lagoons of the Lakshadweep Islands in the Arabian Sea. Botanica Marina 30(1): 83-89.

Richards TA and Cavalier-Smith T (2005) Myosin domain evolution and the primary divergence of eukaryotes. Nature $\mathbf{4 3 6}$ : 1113-1118.

Rogozin IB, Basu MK, Csuros M et al. (2009) Analysis of rare genomic changes does not support the unikont-bikont phylogeny and suggests cyanobacterial symbiosis as the point of primary radiation of eukaryotes. Genome Biology and Evolution 1: 99-113.

Ruiz-Trillo I, Burger G, Holland P et al. (2007) The origins of multicellularity: a multi-taxon genome initiative. Trends in Genetics 23: 113-118.

Ruiz-Trillo I, Inagaki Y, Davis L et al. (2004) Capsaspora owczarzaki is an independent opisthokont lineage. Current Biology 14(22): R946-R947.

Ruiz-Trillo I, Roger AJ, Burger G et al. (2008) A phylogenomic investigation into the origin of Metazoa. Molecular Biololgy and Evolution 25: 664-672.

Schierwater B, Eitel M, Jakob W et al. (2009) Concatenated analysis sheds light on early metazoan evolution and fuels a modern "urmetazoon" hypothesis. PLoS Biology 7: e20.

Sebé-Pedrós A, Roger A, Lang F et al. (2010) Ancient origin of the integrin-mediated adhesion and signaling machinery. Proceedings of the National Academy of Sciences of the USA 107: $10142-10147$.

Shalchian-Tabrizi K, Minge M, Espelund M et al. (2008) Multigene phylogeny of choanozoa and the origin of animals. PLoS One 3: e2098.

Sperling EA, Peterson KJ and Pisani D (2009) Phylogenetic-signal dissection of nuclear housekeeping genes supports the paraphyly of sponges and the monophyly of Eumetazoa. Molecular Biology and Evolution 26: 2261-2274.

Srivastava M, Begovic E, Chapman J et al. (2008) The Trichoplax genome and the nature of placozoans. Nature 454: 955-960.

Steenkamp ET, Wright J and Baldauf SL (2006) The protistan origins of animals and fungi. Molecular Biology and Evolution 23: 93-106.

Stibbs HH, Owczarzak A, Bayne CJ et al. (1979) Schistosome sporocyst-killing Amoebae isolated from Biomphalaria glabrata. Journal of Invertebrate Pathology 33: 159-170.

Tong SM (1997) Heterotrophic flagellates and other protists from Southampton Water, UK. Ophelia 47(2): 71-131.

Torruella G, Suga H, Riutort M et al. (2009) The evolutionary history of lysine biosynthesis pathways within Eukaryotes. Journal of Molecular Evolution 69: 240-248.

Whittaker RH (1959) On the broad classification of organisms. Quaterly Review of Biology 34: 210-226.

Woese CR and Fox GE (1977) Phylogenetic structure of the prokaryotic domain: the primary kingdoms. Proceedings of the National Academy of Sciences of the USA 74: 5088-5090.

Worley AC, Raper KB and Hohl M (1979) Fonticula alba: a new cellular slime mold (Acrasiomycetes). Mycologia 71(4): 746760 . 


\section{Further Reading}

Ruiz-Trillo I, Lane CE, Archibald JM and Roger AJ (2006) Insights into the evolutionary origin and genome architecture of the unicellular opisthokonts Capsaspora owczarzaki and Sphaeroforma arctica. Journal of Eukaryotic Microbiology 53: 1-6.

Steenkamp ET and Baldauf SL (2004) Origin and evolution of animals, fungi and their unicellular allies (Opisthokonta). In:
Hirt RP and Horner DS (eds) Organelles, Genomes and Eukaryote Phylogeny: An Evolutionary Synthesis in the Age of Genomics, pp. 109-129. Boca Raton: CRC Press.

Valentine JW (2004) On the Origin of Phyla. Chicago and London: The University of Chicago Press. 